\title{
Levels of antiviral therapy
}

\begin{abstract}
The problem of the treatment and prevention of acute infectious diseases, especially viral remains relevant for practical health care, despite the wide variety of currently available drugs in the pharmaceutical market. The processes caused by a viral infection largely determine the nature of the flow of infectious process and its outcome. The degree of viremia directly correlates with the manifestations of toxicity, a fever, a reaction of the immune system and the severity of the patient's condition. An important biological feature of viruses is the ability to persistence in the organism and the development of chronic or latent viral infection. The persistence of viruses causes defects of the immune reactivity and creates favorable conditions for the bacterial infection. Thus the priority in the selection of antiviral drugs is at the polling suppression of virus reproduction without significant disturbances of vital activity to host cells. The article describes main levels of antiviral therapy. This review summarized information on the antiviral preparations currently used in clinical as well as in experimental settings. It is shown that the effect of antiviral preparations is possibly at any stage of viral replication.
\end{abstract}

Keywords: Viral replication, Antiviral therapy, ACV, Stages of replication
Volume 3 Issue 3 - 2016

\section{AP Bogoyavlenskiy, AS Turmagambetova, VE Berezin \\ Institute of microbiology and virology, Kazakhstan}

Correspondence: Andrey Pavlinovich Bogoyavlenskiy, Head of Laboratory of Antiviral Protection of Institute of Microbiology and Virology, I03, Bogenbai Batyr Street, Almaty, 0500 I0, Kazakhstan, Tel 7 (777) 3523824, Fax 7 (727) 2918496, Email anpav_63@mail.ru

Received: May 19, 2016 | Published: June 01, 2016
Abbreviations: Ara-A, Vidarabine; ACV, Acyclovir; BVDU, Bromine-5-Vinyl-2'deoxyuridine; IDU, Idoxuridine; TFT, Trifluorothymidine; CMV, Cytomegalovirus; HSV, Herpes Simplex Virus; SARS, Severe Acute Respiratory Syndrome; NA, Neuraminidase; Who, World Health Organization

\section{Introduction}

Infectious diseases are among of the three main causes of people and animals death. Annually, about 2 billion of people worldwide fall ill, more than 17 million of them is dying. ${ }^{1,2}$ Viral infections comprise not less than $60 \%$ of all cases of infectious diseases of humans and animals. The number of viral infections possessing a serious threat to human health and life, as well as causing a considerable economic damage, is not reduced and even increased in recent years. The most striking examples are infectious outbreaks of Ebola, West Nile fever, Sabia fever, Zika virus, MERS, as well as the emergence of new previously unknown diseases caused by viruses of birds, animals and protists. ${ }^{3-7}$ Consequently the study of fundamental mechanisms of resistance to viral infections and development of effective means to prevent and treatment of viral infections remains relevant to this day. ${ }^{8-10}$

The basis of antiviral therapy is the exposure to the virus or its components at any reproduction stage. The process of virus reproduction can be conventionally divided into three phases. ${ }^{8}$ The first phase covers the events that lead to the virus adsorption and penetration into the host cell, the uncoating of the viral core and such viral modifications that is essential for viral infectivity. The second phase of reproduction involves complex processes during which the viral genome is expressed. The final phase of reproduction is the assembly and budding at the host cell's plasma membrane or by lysis, in the latter case, the cell dies. ${ }^{11}$ The arsenal of antiviral drugs available today allows having an impact on any of the stages of virus reproduction.

\section{Viral adsorption and penetration}

The virus-cell interaction begins with the adsorption process, i.e. attachment of viral particles to the surface of host cell. Binding of virus to the cell occurs in two phases: one of which is nonspecific and the other specific. Therefore, the chemotherapy of this stage of infection may also be divided into specific and nonspecific. Nonspecific stage of blocking of virus adsorption is primarily related to the electrostatic interactions between the virus and the cell. Thus the use of polyanionic compounds such as polysulfates, polysulfonates, polycarboxylates, polyhydroxymetalate, polynucleotides and negatively charged albumin or peptides effectively inhibit the adsorption of viruses. ${ }^{12-14}$ Another equally interesting group of chemical compounds that suppress the adsorption of viruses can be called triterpene saponins and steroids. These compounds are capable of changing the spatial structure of the cell membrane because of its structural features by binding with membrane cholesterol..$^{15,16}$

Another important group of nonspecific inhibitors are interferons and their inductors. Interferons trigger multiple secondary processes that can suppress almost all stage of viral replication by binding with cell receptors. ${ }^{17}$

Numerous variations of receptor analogs allowing to "divert" of viral particle from the surface of cells and virus-specific antibodies which bind to the viral receptor can be carried to specific factors of blocking of virus adsorption.

\section{Uncoating of the viral core}

A complex transformation of the virus occurs after penetration of the virion into the host cell. Sense of which is to remove the virus protective covers that prevent the expression of the viral genome. A number of unique features accompanies this process: virus infectivity disappears, sensitivity to nucleases appears, resistance to the action of neutralizing antibodies occurs. The adamantane analogues are the main group of chemical compounds able to inhibit the virus reproduction at this stage. ${ }^{18}$

Adamantane is tricyclic hydrocarbon $(\mathrm{C} 10 \mathrm{H} 16)$. Molecule of adamantane consists of three cyclohexane rings. ${ }^{19}$ The adamantane is not used currently, but the possibility of introducing in position 1 or 3 of different substituents making it promising for antiviral therapy. ${ }^{20}$

Hydrochloride 1-aminoadamantane was introduced in the medical practice as an antiviral agent already in 1966. In medicine this compound is often called midantan or amantadine but the brand name 
-Symmetrel. Mechanism of action of aminoadamantanes consists in the fact that they "interfere" in the early stages of viral reproduction. Their inhibitory effect associated with latency period, start of the replication and synthesis of virus-specific RNA. ${ }^{18}$ This process occurs by blocking of the M2 proton channel in the viral envelope during the step of uncoating of viral particles. Blocking of the M2 channel prevents the changing of medium $\mathrm{pH}$ in endosome and the further process of viral replication.

\section{Expression of viral genomes and assembly of new viral particles}

This stage of virus reproduction is the most complex and consists of: the transcription of nucleic acid, protein translation, posttranslational modifications of viral proteins, replication of nucleic acids and assembly of new virus particles. This stage of reproduction of viruses successfully blocked by 3 major groups of compounds: nucleotide analogs, inhibitors of RNA polymerase and inhibitors of post-translational modifications of proteins.

The mechanism of action of nucleotide analogues consists in the fact that they inhibit the synthesis of nucleic acids according to the principle of anti-metabolism. The action of the most of these compounds in the cell begins at the step of phosphorylation of virusspecific thymidine kinase (herpes and pox viruses) which has a higher affinity to nucleotide analogues than thymidine. The nucleoside analogues include both pyrimidine and purine analogues: vidarabine (Ara-A), aciclovir (ACV), bromine-5-vinyl-2'deoxyuridine (BVDU), idoxuridine (IDU), trifluorothymidine (TFT) and some others.

The antiviral drug ACV is the most effective in treating of herpes simplex virus (HSV) type 1 and 2 as well as in the case of cytomegalovirus (CMV) infection. ${ }^{21}$ Currently a variety of drugsderivatives of ACV approved for the therapeutic uses. Valacyclovir is used in the case of the prevention of CMV infection and the treatment of HSV. Ganciclovir is used to the treat both generalized and local infections, for example, in the case of CMV rhinitis. ${ }^{21,22}$ Penciclovir is a similar in structure with ganciclovir, differs by the replacing of methylene bridge to the ether oxygen in the acyclic moiety of the molecule of ribose. Its metabolism and mechanism of action is similar to ACV. The famciclovir drug is diacetyl 6-deoxy analogue of penciclovir. It's used for the suppression of reproduction of strains resistant to $\mathrm{ACV}^{21}$

The antiviral drug ribavirin is an analogue of guanosine, and has the incomplete purine ring. After the step of intracellular phosphorylation ribavirin-triphosphate inhibits the early stages of mRNA viral transcription (capping and elongation) and thus inhibits the synthesis of ribonucleoprotein. Ribavirin 5'-triphosphate inhibits the activity of enzyme that synthesizes viral RNA polymerase. Ribavirin has a wide spectrum of in vitro activity. ${ }^{23}$ Effectively inhibit such infectious diseases as Lassa fever, Hantavirus, hepatitis $\mathrm{C}$ virus, influenza viruses $\mathrm{A}$ and $\mathrm{B}$, parainfluenza virus, respiratory syncytial virus and measles. ${ }^{24}$ Ribavirin is the only antiviral drug for the treatment of respiratory syncytial virus bronchitis and pneumonia. ${ }^{25}$ Also, the drug was effective in treating of hemorrhagic fevers variety caused by Bunyaviridae. ${ }^{25}$ There are reports on the use of ribavirin in the treatment of viral encephalitis..$^{25}$

According to American virologists, ribavirin effectively inhibits the formation of the specific mRNA structure of the poxviruses family. ${ }^{25}$ Application area of ribavirin as a broad-spectrum of antiviral drug was unexpectedly extended due to the emergence in 2003 of a new infection - severe acute respiratory syndrome (SARS). The basis of treatment of this severe pathology type is oral application of massive daily doses of ribavirin in combination with $\alpha$-2-interferon. ${ }^{22,25}$ Another analogue of nucleotide is pyrimidine nucleoside lamivudine. It's effective against retroviral infections. Lamivudine is an antiretroviral medication used to monotherapy for the treatment of chronic hepatitis $\mathrm{B}$ and in combination with other drugs in the treatment of HIV..$^{22,26}$

Many viral proteins need the post-translational modifications, one of which is the processing. Processing is the proteolytic cleavage, including with the help of host proteases. During this process the viral proteins (especially glycoproteins) acquire the functional activity only after their cutting at specific sites. As a result of such cutting the active form of attachment and fusion proteins arises. A result of this viral particles gain infectious activity. Few effective retroviral protease inhibitors (saquinavir, ritonavir, indinavir, nelfinavir) have been studied in in vivo experiments. ${ }^{27}$ The mechanism of action of these drugs is associated with the formation of acyl-enzyme derivatives. It's based on the blocking of one of the three areas that are critical to the activity of aspartic proteases of the virus: the catalytic core of the enzyme, containing the pair of Asp-Thr/Ser-Gly triad; mobile flap-domain and dimerization domain on the $\mathrm{N}$ - and $\mathrm{C}$-termini of the protease. ${ }^{27,28}$

The protease of other RNA and DNA viruses is an important enzyme and a potential target for antiviral drugs. The difficulty of the obtaining of inhibitors of this enzyme is due to the geometry of the catalytic site and the extended area of interaction with the substrate. However, there were reports of some groups of chemical compounds able to block the enzyme. The group of monocyclic $\beta$-lactam inhibitors (oxazolines and imidazolines) acts by the acylation of the active site of serine to form a stable acyl-enzyme product and blocking a virus protease..$^{29,30}$

\section{Release of new viral particles}

The leave of virions from infected cells is a final stage of the reproduction of virus. This process may occur via budding of virus or cell lysis. The cell lysis is usually accompanied by the cell death. Such mechanism of viral release is practically impossible to block.

Viruses with a lipoprotein envelope realize the escape from the cell by budding. Using this method the cell can remain viable for a long time and produce many thousands of new virus particles until complete exhaustion of its resources. The use of inhibitors of the enzymatic activity of virus involved in virus budding is one of the mechanisms for blocking of this step of viral replication. Developing of the inhibitors of the viral enzyme neuraminidase (NA) is a successful target achievement of modern science - the "rational drug design". Contemporary knowledge of the tertiary structure of NA as the antigen and the enzyme has been used at the synthesis. ${ }^{31}$

Drugs with anti-neuraminidase action "mimic" the structure of the natural substrate of the catalytic site of NA, which "attracts" the virus to more interaction with them. ${ }^{32}$

The first official reports of preparations with anti-neuraminidase action were made by $\mathrm{M}$ von Itzstein et al. in 1993 (zanamivir) and Kim CU et al. in 1997 (oseltamivir). ${ }^{32}$ Their practical application was started in 1999. Oseltamivir quite quickly gained the right to be the drug of choice for the treatment of influenza infection during the epidemics and to create a reserve in case of a pandemic. ${ }^{1}$ WHO experts have added recommendations by inclusion zanamivir in the list of in preparation for a pandemic, because of the emergence of resistant influenza viruses in a population of epidemic strains of A (H1N1), as well as among the highly pathogenic avian influenza virus strains $\mathrm{A}(\mathrm{H} 5 \mathrm{~N} 1){ }^{33}$ 
Zanamivir is administered directly to the respiratory tract in the form of a fine powder using an inhaler. It is not metabolized. The active substance distributed at high concentrations up to $90 \%$ in the upper respiratory tract and from 10 to $20 \%$ in the lung. ${ }^{31,32}$

\section{Conclusion}

Actually practical medicine has a quite impressive arsenal of antiviral agents capable of suppressing of virus replication at any stage. However, despite of some progress achieved in viral chemotherapy the clinical practice faces with a serious problem of appearance of virus variants that are resistant to certain drugs. Thus the efficiency of adamantane and its derivatives is currently limited to emerging of resistant strains of influenza A virus. Formation of strains resistant due to the replacement of amino acid on positions $26,27,30,31$ and 34 for the part of M2 transmembrane channel. ${ }^{8,31,34}$ The analysis of more than 60,000 strains of influenza A virus (H3N2), isolated in various countries over the last decade, showed increased resistance to the M2channels inhibitors with $0.4 \%$ in 1995 year to $89.3 \%$ in 2013 year The frequency of resistance was the highest in Asia and in Central Africa. There were $96.8 \%$ resistant isolates in China in 2008 and $100.0 \%$ resistant isolates in Kenya and Uganda. ${ }^{35} \mathrm{~A}$ high frequency of resistance to amantadine of influenza A isolates was also observed in the United States at season 2012-2013. Thus $93.2 \%$ of the strains of influenza A virus (H3N2) and $88.9 \%$ of the strains of influenza A virus (H1N1) had the amino acid substitution on position 31 of M2-channel gene. A similar frequency of resistance to the influenza A virus $\mathrm{H} 3 \mathrm{~N} 2$ (100\% in 2010) was observed during this period in Canada. ${ }^{2,35}$

It was previously thought that the development of resistance to the influenza virus neuraminidase inhibitors is rare and is not a clinical problem. The frequency of resistance of clinical isolates of influenza A virus does not exceed $1.5 \%$ in 2001 . The resistance to oseltamivir was $0.32 \%$ and $4.1 \%$ for children and adults, respectively. Drug resistance strains were not found among the strains of influenza B virus. ${ }^{8}$ However the recent data suggest the development of resistance to the neuraminidase inhibitors from 0 to $64 \%$ of isolates of influenza $\mathrm{A}$ and $\mathrm{B}$ viruses in different countries. ${ }^{36}$

The viruses can avoid the mechanism of action such drug as inhibitors of viral proteases action after a few replication cycles. ${ }^{27,37}$

The study of plant medicines that have anti-viral properties (alpisarin, epigen, proteflasid and gossypol) showed that due to the different mechanisms of action (antiviral and immunostimulatory), the emergence of viruses resistant to these drugs is not observed. A wide variety of biologically active compounds was found in the plants growing on the territory of Kazakhstan. It's possible to obtain the new highly active drugs with the ability to block a variety of viruses from such plants, including those that are resistant to existing commercial chemical preparations from similar preparations which combine immunostimulatory and antiviral properties can be recommended for the treatment and prevention of infectious diseases associated with immunodeficiency, including influenza, HIV, hepatitis C and others. $^{38-40}$

Peculiarities of life cycle of viruses as intracellular parasites at the molecular-genetic level make it necessary to constant search for the substances that have the ability to suppress the viral replication and spread.

\section{Acknowledgments}

None.

\section{Conflicts of interest}

None.

\section{References}

1. Thayer AM, Houston CEN. Flu fighters. Chem Eng News. 2009;87(39):15-26.

2. World Health Organization. World Health Statistics. WHO. 2015.

3. Musso D, Aubry M, Broult J, et al. Zika virus: new emergencies, potential for severe complications, and prevention of transfusiontransmitted Zika fever in the context of co-circulation of arboviruses. Blood Transfus. 2016;28:1-2.

4. Rasmussen AL, Katze MG. Genomic Signatures of Emerging Viruses: A New Era of Systems Epidemiology. Cell Host Microbe. 2016;19(5):611-618.

5. Al-Tawfiq JA, Omrani AS, Memish ZA. Middle East respiratory syndrome coronavirus: current situation and travel-associated concerns. Front Med. 2016

6. Shao J, Liang Y, Ly H. Human hemorrhagic Fever causing arenaviruses: molecular mechanisms contributing to virus virulence and disease pathogenesis. Pathogens. 2015;4(2):283-306.

7. Ong CW. Zika virus: an emerging infectious threat. Intern Med $J$. 2016;46(5):525-530.

8. Preziosi P. Influenza pharmacotherapy: present situation, strategies and hopes. Expert Opin Pharmacother. 2011;12(10):1523-1549.

9. Boltz DA, Aldrige JR, Webster RG, et al. Drugs in development for influenza. Drugs. 2010;70(11):1349-1362.

10. Beigel J, Bray M. Current and future antiviral therapy of severe seasonal and avian influenza. Antiviral Res. 2008;78(1):91-102.

11. Krug RM, Aramini JM. Emerging antiviral targets for influenza A virus. Trends Pharmacol Sci. 2009;30(6):269-277.

12. Bergstrom DE, Lin X, Wood TD, et al. Polysulfonates derived from metal thiolate complexes as inhibitors of HIV-1 and various other enveloped viruses in vitro. Antivir Chem Chemother. 2002;13(3):185-195.

13. Sepúlveda-Crespo D, Gómez R, De La Mata FJ, et al. Polyanionic carbosilane dendrimer-conjugated antiviral drugs as efficient microbicides: Recent trends and developments in HIV treatment/ therapy. Nanomedicine. 2015;11(6):1481-1498.

14. Tan S, Lu L, Li L, et al. Polyanionic candidate microbicides accelerate the formation of semen-derived amyloid fibrils to enhance HIV-1 infection. PLoS One. 2013;8(3):e59777.

15. Xiao S, Si L, Tian Z, et al. Pentacyclic triterpenes grafted on CD cores to interfere with influenza virus entry: A dramatic multivalent effect. Biomaterials. 2016;78:74-85.

16. Li Y, Jiang R, Ooi LS, et al. Antiviral triterpenoids from the medicinal plant Schefflera heptaphylla. Phytother Res. 2007;21(5):466-470.

17. Loginova SY, Koval'chuk AV, Borisevich SV, et al. Antiviral activity of an interferon inducer amixin in experimental West Nile Fever. $\mathrm{Vopr}$ Virusol. 2004;49(2):8-11.

18. Cady SD, Schmidt-Rohr K, Wang J, et al. Structure of the amantadine binding site of influenza M2 proton channels in lipid bilayers. Nature. 2010;463(7281):689-692.

19. Balfour HH. Antiviral Drugs. New Engl JMed. 1999;340(16):1255-1268

20. Spasov AA, Khamidova TV, Bugaeva LI, et al. Adamantane Derivatives: Pharmacological And Toxicological Properties (Review). Pharmaceutical Chemistry Journal. 2000;34(1):1-7. 
21. Sidwell R, Huffman J, Barnard M, et al. Effect of phosphonic acid analogs of acyclovir and gancyclovir on in vitro cytomegalovirus infections. Nucleotides and Nucleosides. 1989;8(5-6):833-836.

22. Galegov GA, Andronova VL, Leont'eva NA, et al. Etiotropic medicinal therapy of viral infections. Vopr Virusol. 2004;49(3):35-40.

23. Deyrup M, Sidwell R, Little R, et al. Improved delivery through biological membranes. Synthesis and antiviral activity of a series of ribavirin chemical delivery systems: 2' and 3' derivatives. Antiviral Chem and Chemother. 1991;2(6):337-355.

24. Sidwell RW. Ribavirin: a review of antiviral efficacy. Antimicrobial Agents \& Chemotherapy. 1996;1:219-255.

25. Stephen EL, Jones DE, Peters CJ, et al. Ribavirin treatment of Toga-, Arena- and Bunyavirus infections in subhuman primates and other laboratory animal species. In: RA Smith \& W Kirkpatrick (Eds.), Ribavirin: A broad spectrum antiviral agent, New York: Academic Press, Inc., New York, USA. 1980;pp.169-183.

26. Sriprayoon T, Mahidol C, Ungtrakul T, et al. Efficacy and safety of entecavir versus tenofovir treatment in chronic hepatitis B patients: A randomized controlled trial. Hepatol Res. 2016.

27. Patick AK, Potts KE. Protease inhibitors as antiviral agents. Clinic Microbiol Rev. 1998;11(4):614-627.

28. Tretyakova EV, Smirnova IE, Salimova EV, et al. Synthesis and antiviral activity of maleopimaric and quinopimaric acids' derivatives. Bioorg Med Chem. 2015;23(20):6543-6550.

29. Visalli RJ, Ziobrowski H, Badri KR, et al. Onic derivatives of betulinic acid exhibit antiviral activity against herpes simplex virus type-2 (HSV-2), but not HIV-1 reverse transcriptase. Bioorg Med Chem Lett. 2015;25(16):3168-3171.
30. Kishbaugh TL. Pyridines and Imidazopyridines With Medicinal Significance. Curr Top Med Chem. 2016.

31. Hayden FG. Antiviral for influenza: historical perspectives and lessons learned. Antiviral research. 2006;71(2-3):372-378.

32. Bantia S, Parker CD, Ananth SL, et al. Comparison of the anti-influenza virus activity of RWJ-270201 with those of oseltamivir and zanamivir. Antimicrob. Agents and Chemother. 2001;45(4):1162-1167.

33. Yusuf M, Mohamed N, Mohamad S, et al. H274Y's Effect on Oseltamivir Resistance: What Happens Before the Drug Enters the Binding Site. J Chem Inf Model. 2016;56(1):82-100.

34. Jacob A, Sood R, Chanu KhV, et al. Amantadine resistance among highly pathogenic avian influenza viruses (H5N1) isolated from India. Microb Pathog. 2016;91:35-40.

35. Dong G, Peng Ch, Luo J, et al. Adamantane-Resistant Influenza A Viruses in the World (1902-2013): Frequency and Distribution of M2 Gene Mutations. PLoS One. 2015;10(3):e0119115.

36. Khodadad N, Moattari A, Shamsi Shahr Abadi M, et al. Prevalence of Influenza A(H1N1)pdm09 Virus Resistant to Oseltamivir in Shiraz, Iran, During 2012-2013. Jundishapur J Microbiol. 2015;8(8):e23690.

37. Ferraris O, Lina B. Mutations of neuraminidase implicated in neuraminidase inhibitors resistance. J Clin Virol. 2008;41(1):13-19.

38. Shen Z, Lou K, Wang W. New small-molecule drug design strategies for fighting resistant influenza A. Acta Pharm Sin B. 2015;5(5):419-30.

39. Fabricant DS, Farnsworth NR. The value of plants used in traditional Medicine for drug discovery // Environmental Health Perspectives. 2001;109(Supp11):69-75.

40. Safrin S. Antiviral agents. In: Katzung BG (Edr.), Basic and clinical pharmacology, McGrow-Hill, New York, USA. 2007;pp.815. 